\title{
A pursuit of lineage-specific and niche-specific proteome features in the world of archaea
}

\author{
Anindya Roy Chowdhury and Chitra Dutta*
}

\begin{abstract}
Background: Archaea evoke interest among researchers for two enigmatic characteristics -a combination of bacterial and eukaryotic components in their molecular architectures and an enormous diversity in their life-style and metabolic capabilities. Despite considerable research efforts, lineage- specific/niche-specific molecular features of the whole archaeal world are yet to be fully unveiled. The study offers the first large-scale in silico proteome analysis of all archaeal species of known genome sequences with a special emphasis on methanogenic and sulphur-metabolising archaea.

Results: Overall amino acid usage in archaea is dominated by GC-bias. But the environmental factors like oxygen requirement or thermal adaptation seem to play important roles in selection of residues with no GC-bias at the codon level. All methanogens, irrespective of their thermal/salt adaptation, show higher usage of Cys and have relatively acidic proteomes, while the proteomes of sulphur-metabolisers have higher aromaticity and more positive charges. Despite of exhibiting thermophilic life-style, korarchaeota possesses an acidic proteome. Among the distinct trends prevailing in COGs (Cluster of Orthologous Groups of proteins) distribution profiles, crenarchaeal organisms display higher intra-order variations in COGs repertoire, especially in the metabolic ones, as compared to euryarchaea. All methanogens are characterised by a presence of 22 exclusive COGs.

Conclusions: Divergences in amino acid usage, aromaticity/charge profiles and COG repertoire among methanogens and sulphur-metabolisers, aerobic and anaerobic archaea or korarchaeota and nanoarchaeota, as elucidated in the present study, point towards the presence of distinct molecular strategies for niche specialization in the archaeal world.
\end{abstract}

Keywords: Amino acid usage, Isoelectric point, COG distribution, Methanogen, Sulphur metaboliser, Korarachaeota, Oxygen requirement

\section{Background}

Over the past few decades, the process of establishing archaea as the third domain of life has been a stunning event in the world of life science. The world became familiar with this kingdom in 1977, when Woese \& Fox [1] first proposed archaebacteria (subsequently renamed archaea) as a major domain - distinct from bacteria and eukaryotes but on equal footing with them. Prior to this three domain classification of life, which has been described by Makarova \& Koonin [2] as "arguably one of the most important scientific discoveries of the twentieth century"; many of the 'would-be' archaea, used to be

\footnotetext{
* Correspondence: cdutta@iicb.res.in

Structural Biology \& Bioinformatics Division, CSIR Indian Institute of Chemical Biology, 4, Raja S. C. Mullick Road, Kolkata 700032, India
}

grouped under the bacterial lineage [2-4]. Phylogenetic analyses of rRNA and some proteins involved in the processes of translation, transcription, and replication have placed the notion of archaea on a firm footing [5-9]. Analysis of small subunit rRNA sequences revealed that there are two distinct phyla viz. euryarchaeota and crenarchaeota within this third domain [10]. Three more distinguished phyla viz. nanoarchaeota, korarchaeota and thaumarchaeota have later been introduced to the domain of archaea [11-17].

Subsequent work on archaea has revealed a lot of surprises that have invoked an urge in the scientific community to explore the world of these microbial life forms. Archaea have a unique mosaic combination of "eubacterial form and eukaryotic content". Like bacteria, they are single- celled prokaryotes, devoid of nucleus or

\section{( Biomed Central}


other cell organelles [18]. They usually share some major aspects of genome organisation and expression strategy such as presence of single circular chromosome and absence of introns, the operonic organisation of certain genes, presence of ribosomal-binding (Shine-Dalgarno) sites and so on; though there are some reports on the presence of archaeal introns $[19,20]$. Yet they possess a number of genes and metabolic pathways - especially the ones associated with the processes of transcription, translation and replication - typical of eukaryotes $[21,22]$. More than 30 ribosomal proteins are shared between the archaea and the eukarya that are not found in the bacteria. The structure of chromatin, presence of histones, significant similarity between proteins involved in information processing systems - all indicate a close evolutionary link between archaea and eukaryotes [23-26]. Archaea also possess some unique characteristics not shared by other domains. For example, their membrane is made of ether linked lipids. The glycerol phosphate backbone has got an opposite stereochemistry as compared to bacteria or eukaryotes $[27,28]$. They also exhibit some unique metabolism like methanogenesis and several unique enzymes e.g. specific types of DNA topoisomerases and DNA polymerases [29-31]. Till date there has been no report on archaeal virulence, but they have been found associated with the diseased state of colon and periodontal diseases [32,33].

Another intriguing feature of archaea is their unusual ability to survive and thrive in the extreme environmental conditions, such as in thermal vents, volcanic springs, hypersaline basins, alkaline lakes, acid mines, or even in petroleum deposits at deep underground which is completely devoid of oxygen. Furthermore, certain groups of archaea employ distinct strategies for energy conversion and hence, are characterised by special metabolic traits like methane production under anaerobic conditions, or sulphur respiration. Adaptation to such extreme environment or to atypical metabolism is expected to require special, adaptive gene and/or protein features - clearly distinguishable from those of the organisms living under the conventional ecological conditions. There are some reports on the molecular, physiological and evolutionary mechanisms of adaptation of some specific groups of extremophilic microbes, including some archaea, such as the organisms adapted to high temperature or salinity [34-39].

But to our knowledge, no comprehensive comparative study on lineage-specific and/or niche- specific genome/proteome features of the archaeal world has so far been reported.

Therefore, the domain archaea seems a deep sea where the researchers can dive into to get more and more information about their specific characteristics. Availability of complete genome sequences of hundreds of archaea has paved a way for comparative genomics and proteomics study. The lack of established model systems for large-scale experimentation on archaeal biology has made in-silico genome data mining even more crucial for archaeal genomics than they are in the cases of bacteria and eukaryotes. The present analysis offers the first large-scale comparative study of the proteomic architectures of all the archaeal species of publicly available genome sequences. Special emphasis has been given on the comparative analysis of methanogenic and sulphur metabolising archaea with an aim to unveil the special nichespecific molecular features, if any, of these two groups of microbes with specialised life-style. Identification of such features may not only give an insight into the molecular mechanism of ecological adaptation in archaea, but may also be important from the metagenomic or biotechnological view-points.

\section{Results and discussion}

\section{Analysis of the whole archaeal dataset} Amino acid usage profile within the groups

Figure 1 depicts the complete linkage clustering of 69 archaeal organisms under study on the basis of the average frequencies of occurrences of different amino acid residues in the respective proteomes (for organism details, see Additional file 1). The left panel of the figure represents a heat map of the relative amino acid usage values, where the colour gradient from red to green shows the increment in the respective values in a particular column i.e. for a particular amino acid residue. The residues like Tyr, Lys, Asn, Ile and Phe, encoded with AU-rich codons, are presented in the extreme left side of the heat map, while the residues encoded by GCrich codons, such as Pro, Ala, Arg and Gly are displayed in the extreme right side of the map. The right panel of Figure.1 represents the clustering of the organisms on the basis of their amino acid usage, where the organisms are segregated in two distinct sub-clusters under the nodes $\mathrm{A}$ and $\mathrm{B}$. The organisms clustered under the node $\mathrm{A}$, in most cases, have an average genomic GCcontent $\geq 40 \%$, while those segregated under the node $B$ are marked by relatively less $\mathrm{G}+\mathrm{C}$-bias (average GCcontent $\leq 40 \%$ ), indicating that the amino acid usage in the archaeal species under examination are primarily governed by their genomic $\mathrm{G}+\mathrm{C}$-bias, though certain lineage-specific and/or niche-specific trends in their amino acid usage can also be identified within or even across different clusters. Appearance of red coloured blocks at two diagonally opposite corners of Figure 1, indicating under-representation of the residues encoded by AU-rich codons in organisms with high GC-content and of those encoded by GC-rich codons in GC-poor organisms, also advocates for influence of genomic GCbias on archaeal proteome composition. 


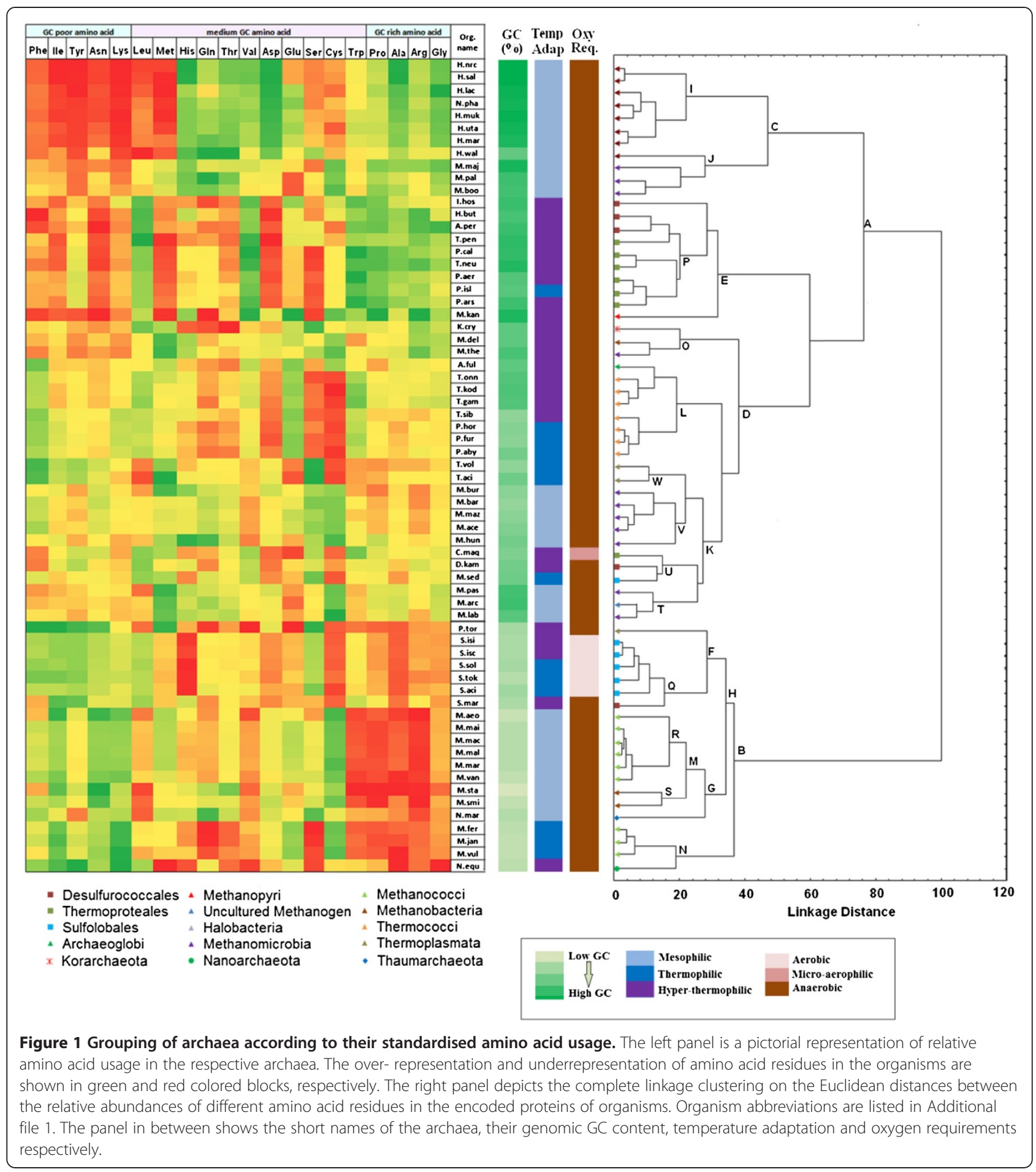

However, a closer look at the heat map reveals that the amino acid preferences by archaeal species are not solely governed by the mutational bias of the respective genomes. Taxonomic or ecological background of the species may also play important roles in shaping their protein composition. In many cases, members of the same phyla, class or order appear under distinct nodes far apart from one another, yet they share some common compositional features, which may not always comply with their genomic GC-bias. For instance, P. torridus (P. tor), a thermoplasmata species, appears under node F, far apart from T. acidophilum (T.aci) and T. volcanium ( $\mathrm{T}$. vol), two other members of thermoplasmata that clustered together under node W. 
But in all these species, the usages of Phe and Met, two residues encoded by AU-rich codons, are higher than sulfolobales, methanococci or nanoarchaeota- the genomic GC-contents of which are comparable to or lower than those of thermoplasmata. These three species are also typified by relatively low usage of Glu, Leu and Cys and higher usage of Ser. The sulfolobales having similar GC-bias as P. tor (35-37\%) in general, segregate together with P. tor under node F, but they differ in the usage patterns of amany of the residues like Leu, His, Asp, Trp etc., and ma ny of these features are also shared by M. sedula (M. Sed) - the only member of sulfolobales in the dataset with much higher GC-content (46\%, under node U). A trend of lower usage of Asp and Met and higher usage of Leu and Val is observed in all thermoprot eales including C. maquilingensis (C. maq) with much lower GC-content (43\%), which has clustered together with $\mathrm{M}$. sed and D. kam - far apart from other thermoproteales (GC-content $>50 \%$ ). Each of the three single-member phyla in the dataset, namely nanoarchaeota, korarchaeota and thaumarchaeota, exhibit distinct trends in amino acid usage and appear as singular species in separate branches under the nodes N, O and G respectively. A detail examination of the biological implications of such conspicuous amino acid usage patterns is, however, beyond the scope of the present study and will be taken up separately in future.

From their proteome compositional features, archaea appear to adapt to specific niche or life- style. Most of the methanogens exhibit relatively high frequencies of Cys. All the halophiles have clustered under node I (except H.wal) and are marked with high usage of Asp, Thr and His and low occurrence of Cys, Leu and Met. It is worth noting that $H$. walsbyi $(\mathrm{H}$. wal) (displayed under node J) has much lower GC-content (48\%) than other halophiles (>60\%), yet it shares many typical features of high salt-adapted proteomes like under representation of Lys, Phe, Tyr, Met and Leu (all of these except Leu are encoded by AU-rich codons), and over representation of Asp, Thr, His etc. It may, therefore, be said that the genomic GC-bias, taxonomic history and life-style or niche adaptation - all have played important roles in sculpting the amino acid composition of an archaeon.

\section{Physico-chemical characteristics of the proteomes of different groups of archaea}

With a view to understand the physico-chemical basis of distinct trends in amino acid composition of archaeal proteomes within and across different lineages, various physico - chemical parameters like mean hydropathy, aromaticity and isoelectric points have been calculated individually for all predicted protein sequences for each of the organisms under study. While the distribution of mean hydropathy and aromaticity values show little variations across the proteomes (data not shown), significant variations have been observed among different groups of archaeal proteomes in distribution of $\mathrm{pI}$ or isoelectric points of predicted proteins. Figure 2 shows the average distribution profiles of the predicted isoelectric points (pI) of the deduced amino acid sequences for different classes/orders of archaeal organisms under study.

We have categorised the organisms according to their classes in case of euryarchaeota (Figure 2a) and down to their orders in case of crenarchaeota (Figure 2b), as the entire crenarchaeal group comes under a single class viz. thermoprotei. The remaining three phyla viz. korarchaeota, nanoarchaeota and thaumarchaeota (Figure 2c), have only one fully sequenced organism in each case, so there is no need of any further division.

In most of the cases, bimodal distributions of isoelectric points are observed with an acidic peak at $\mathrm{pI}$ range of 5.0-5.5 and a basic peak at 9.5 [Here, "acidic peak" refers to the frequency peak in the isoelectric point plot, where the $\mathrm{pI}$ range lies around the acidic $\mathrm{pH}$ region $<$ $\mathrm{pH}$ 7.0, similarly, "basic peak" refers to the region around $\mathrm{pH}>7.0]$. Being the largest phylum in the archaeal world, euryarchaeota consists of eight classes and for all these classes except thermococci, the acidic peak is significantly higher than the basic peak, implying the overall acidic nature of the euryarchaeal proteomes, irrespective of their genomic GC-bias or niche adaptation. Among these, Halobacteria, a group of halophilic archaea, has the most acidic proteome showing a large acidic peak around pI 4.0 and almost no peak at basic pI - a feature attributable to over representation of Asp and under representation of Lys, as observed earlier in most of the microbial halophiles [37,40]. The only methanopyri in the dataset, $M$. kandleri, which is known to have dual adaptation to high salinity and high temperature, also exhibits a large and sharp acidic peak around pI 5.0 along with a small basic peak. A large acidic peak at pI 5.0 is also displayed by methanobacteria. Though their salinity adaptation is not yet reported, they have been found in large amounts in the tropical estuarine sediments along with other halophilies [41]. For all other euryarchaeal proteomes, acidic peaks (at pI values around 6.0) are slightly larger than the respective basic peaks (at pI values around 10.0), implying that these proteomes are also comparatively acidic in nature, whereas thermococci stands out as an exception, probably owing to their adaptation to high temperature and sulphur metabolism.

Crenarchaeal organisms are all under one class viz. thermoprotei. Though we have further divided them into three orders, they do not exhibit any significant variation in their pI profiles. For all three orders, proteomes are comparatively basic in nature (Figure $2 b$ ). 


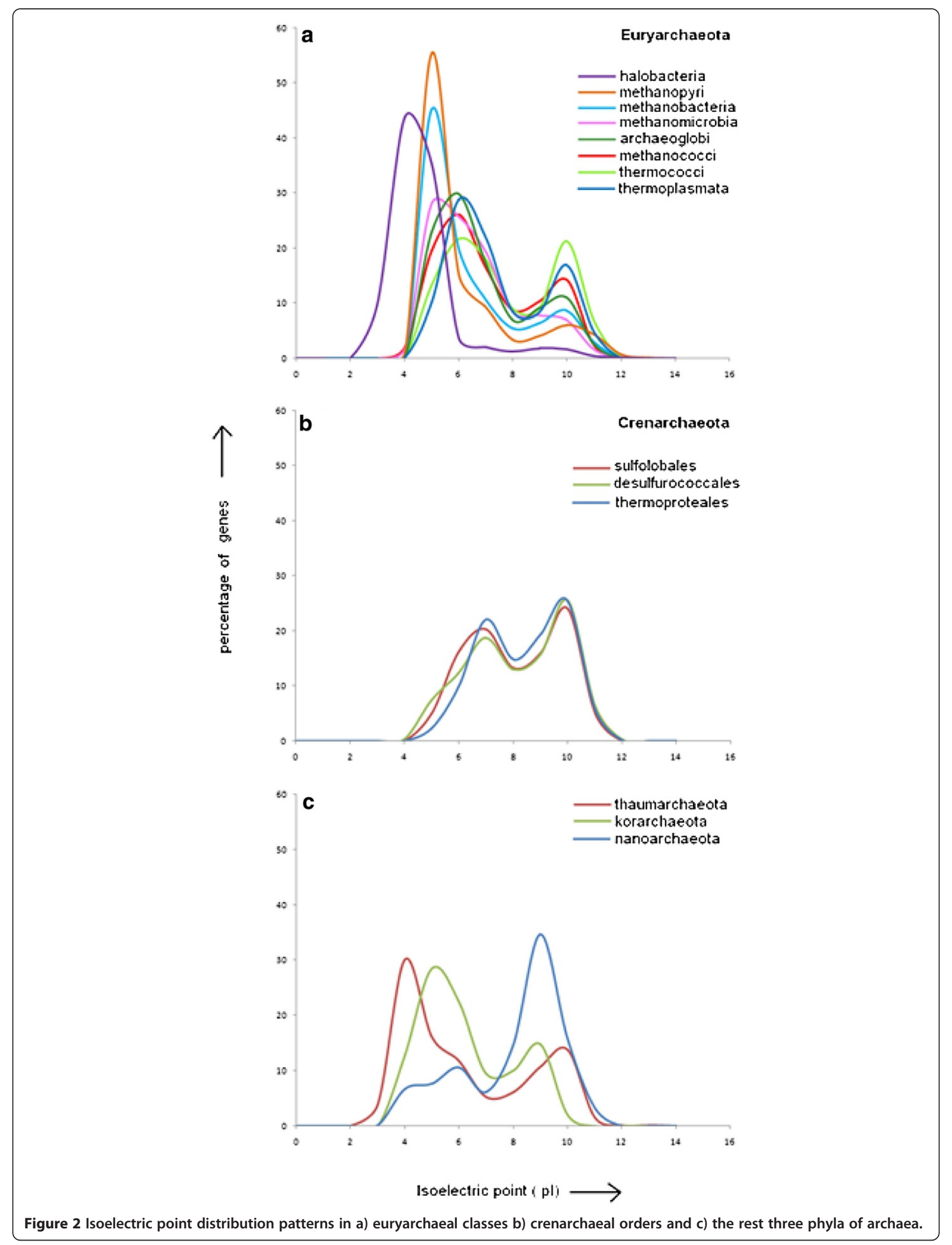


As reported earlier, nanoarchaeota, being a parasitichyperthermophile, has a highly basic proteome, while the proteome of thaumarchaeota, being mesophilic in nature, is comparatively acidic (Figure 2c) [12,34]. Korarchaeota, in spite of being a hyperthermophile, has an acidic proteome, which is quite surprising in view of earlier reports on thermal adaptation of microbial proteomes [42]. However, considering the fact that korarchaeal samples were collected from the Obsidian Pool, Yellowstone National Park [13], the possibility of hypersaline adaptation in K. cryptofilum cannot be ruled out and in that case, the halophilic signatures of its proteome may overshadow the thermophilic characteristics, as observed in M.kandleri.

\section{Distribution of cluster of orthologous groups of proteins}

With a view to assess the similarities and divergences in the genetic make-up of different classes/orders of archaeal species, we took advantage of the COGs functional classification, which is based on orthologous relations among genes [43]. Figure 3 shows a plot of the ratio of normalised genes to total gene content for each COGs category in archaeal genomes of different taxonomic clases/orders, where Escherichia coli and
Saccharomyces cerevisiae have been taken as control representatives of the bacteria and eukaryotic domains.

As revealed in Figure 3, the overall COGs distribution profiles of the archaeal groups are, in general, much closer to that of E. coli than to the Yeast profile. Majority of the COGs categories related to cellular processes \& signaling are present in relatively low frequencies in archaea as compared to the eukaryotic representative $S$. cerevisiae. However, proteins belonging to the categories $M$ (cell wall/memberane/envelope biogenesis) and $\mathrm{N}$ (cell motility) have higher frequencies in archaea as well as in $E$. coli than in yeast. The COGs related to metabolism also have in relatively high frequencies in archaea than in Yeast.

$\mathrm{R} \& \mathrm{~S}$, the two categories of poorly characterised genes, together encompass around 20 to $28 \%$ of predicted gene-products in each group of archaeal proteomes (Figure 3). Among the well characterised COGs categories, the ones showing the highest abundances in distribution profiles across different classes/orders are E (amino acid transport and metabolism), J (translation, ribosomal structure and biogenesis) and $\mathrm{C}$ (energy production and conversion), whereas the categories showing largest standard deviations are J (translation, ribosomal structure and biogenesis), $\mathrm{T}$ (signal transduction

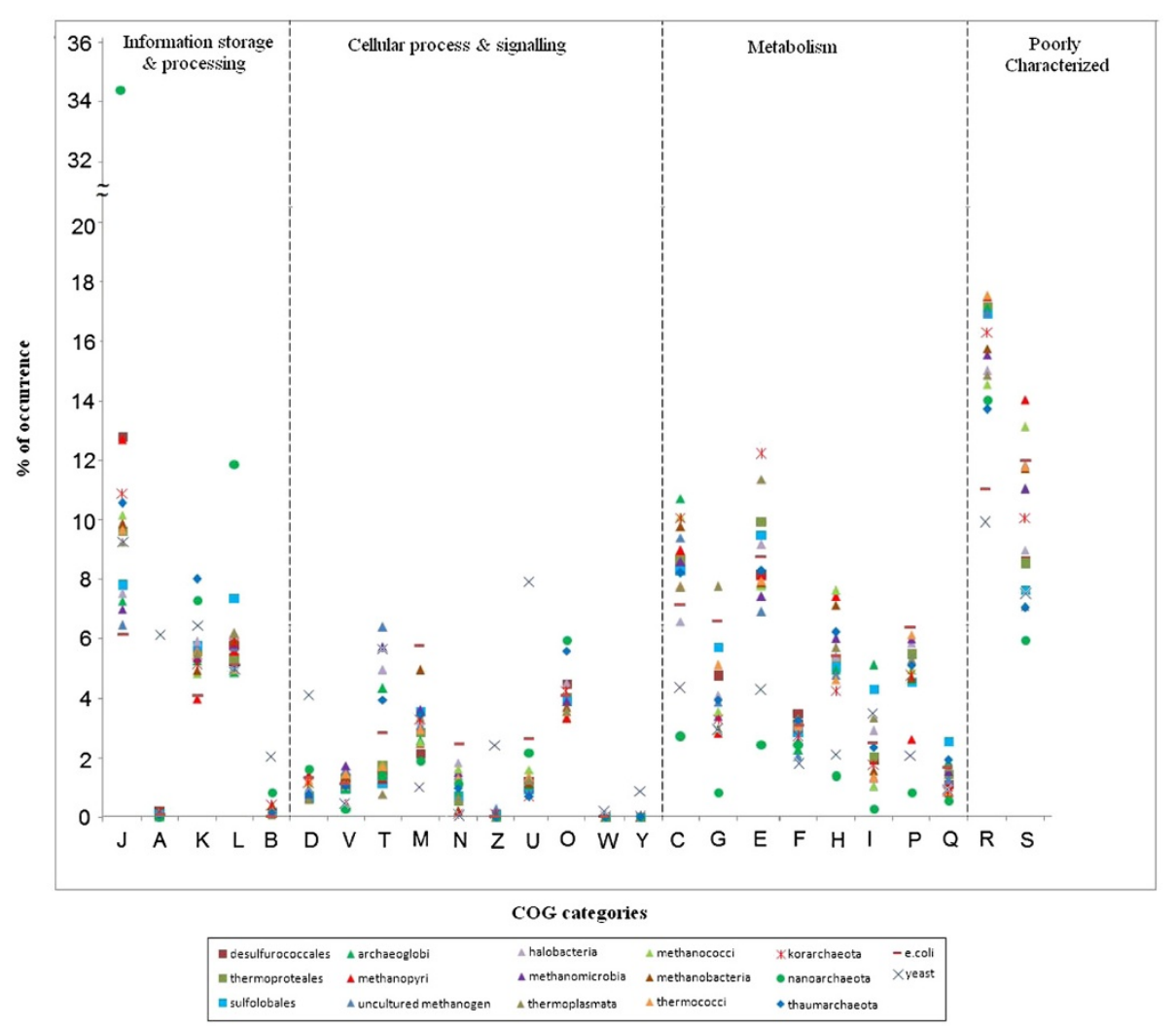

Figure 3 Distribution of COGs categories of the entire archaeal group under study and their comparison with E.coli (member from bacterial kingdom) and yeast (member from eukaryotic kingdom). 
mechanism) and L (replication, recombination and repair). Nanoarchaeota shows a very different COGs distribution pattern with $34.5 \%$ of its total proteome falling under the translation, ribosomal structure and biogenesis (J) category (Additional file 2). Strikingly enough, most of the COGs categories pertaining to metabolism, such as C, G, E, H, I, P and Q are significantly underrepresented in $N$. equitans. Such a conspicuous trend in COGs distribution in $N$. equitans may be attributed to the parasitic/symbiotic lifestyle of the organism. Methanopyri, the halophilic, thermophilic archaeon, is characterised with comparatively low amount of $\mathrm{K}$ (transcription) and P (inorganic ion transport \& metabolism) category.

\section{Detail analysis of methanogenic and sulphur- metabolising archaea}

The dataset of 69 archaeal species under study includes various types of extremophiles - the species thriving in extreme habitats such as thermal vents or hypersaline water as well as the species exhibiting specialised metabolism, such as methanogenesis or sulphur metabolism. Since the distinct genome/proteome features of thermophilic and halophilic organisms have been reported earlier

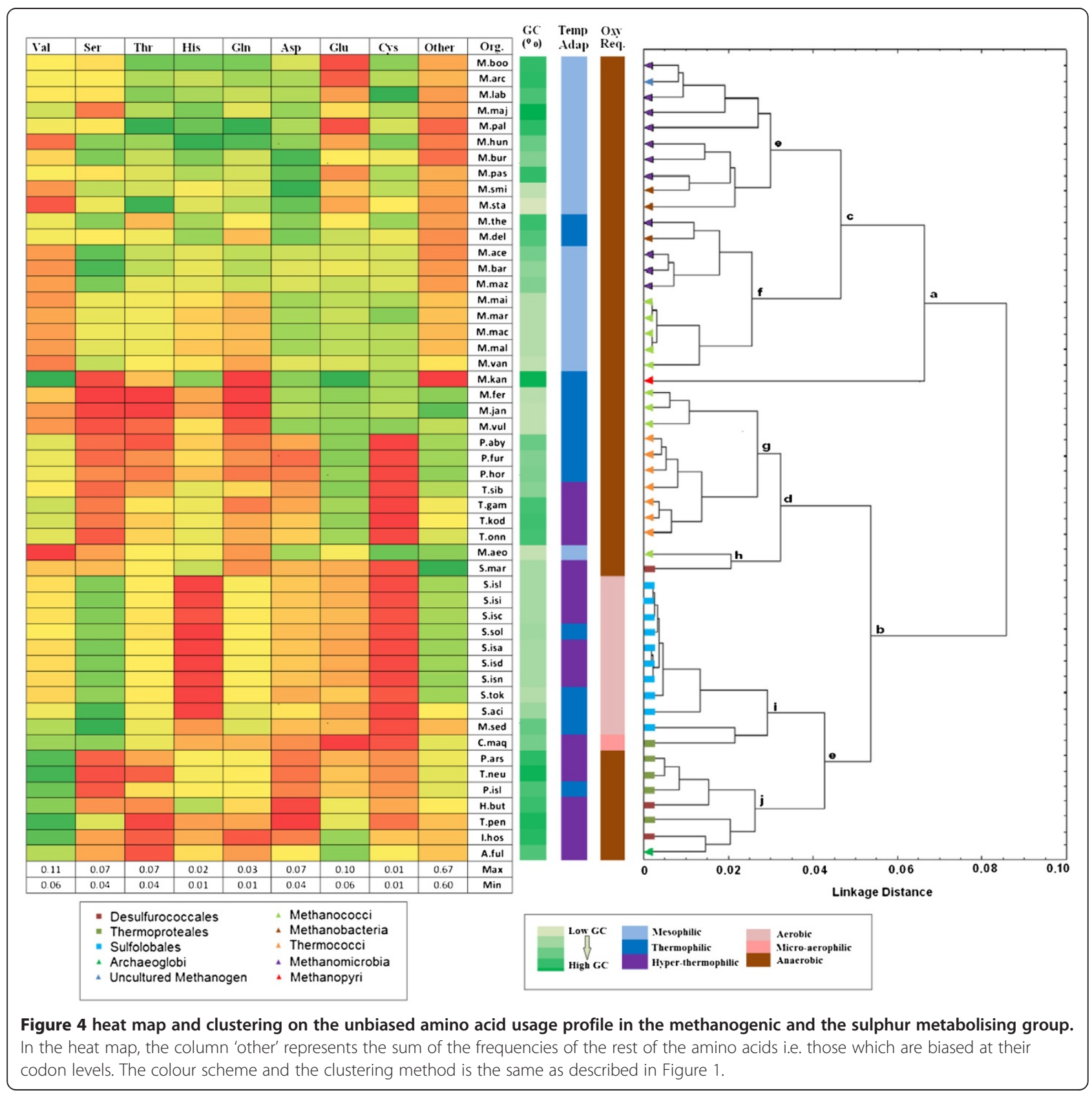


$[44,45]$, an attempt is made in the present study to delineate the niche-specific molecular features, if any, of the groups of archaea exhibiting specialised metabolic traits, i.e., the methane-producing archaea and the sulphuroxidising/sulphur-reducing archaea (for details, see Additional file 3). It is worth mentioning at this point that these two groups of archaea also contain some thermophilic/hyperthermophilic and acidophilic organisms and as already mentioned, $M$. kandleri exhibits dual adaptation to thermophilic and halophilic environments [46].

\section{Usage of amino acids with no GC bias at the codon levels}

The trends in overall amino acid usage of methanogenic and sulphur-metabolising archaea have already been discussed in context of Figure 1, where the organisms are clustered according to their amino acid usage. But as depicted in this figure, the impact of genomic GC-bias on the amino acid usage is so strong that the organisms under study have been segregated, in effect, on the basis of their average genomic GC-content. However, one cannot rule out the possibility of existence of other selection pressures on amino acid usage, influence of which might have been overshadowed by the effect of the GC-bias and hence, could not be detected in Figure 1. In order to identify and characterise such selection pressures, if any, one needs to mask the pronounced effect of the genomic GC-bias in the cluster analysis and heat-map of amino acid usage. To this end, we present Figure 4, where despite of considering all the 20 amino acids, only those are chosen, which have no GC bias in their respective codons, viz. Val, Ser, Thr, His, Gln, Asp, Glu and Cys. The frequencies for the remaining amino acids are taken as a sum during the process of clustering, so that no artifact arises owing to this exercise.

As revealed in Figure 4, the two factors that primarily govern the usage of these eight amino acid residues are the oxygen requirement and temperature adaptation of the respective archaeal species. All aerobic species along with C. maq, the only micro-aerophilic archaeon in the dataset, are completely segregated from the anaerobic organisms and clustered exclusively under the node $\mathrm{i}$, suggesting the significant influence of respiratory habits of the organisms on preferences for these amino acids. Dominance of temperature adaptation of the species is apparent from the observation that there are two major nodes $\mathrm{a}$ and $\mathrm{b}$ in Figure 4, dividing whole set of methanogens and sulphur-metabolisers under consideration into two major clusters, where all mesophiles except $M$ aeolicus have been segregated under the node a, and all hyperthermophiles and majority of the thermophiles (except M. the, M. del and M. kan) have clustered together under the node $b$.

All crenarchaeal species except S. mar are clustered together under the node e. A carefu l examination of the accompanying heat map suggests that the unexpected segregation of S.mar and M.aeo apparently represents an artifact, since this segregation might be attributed to the similarity in the "others" column, which represents total frequencies of occurrence of the residues encoded by GC-rich/AU-rich codons.

In the heat map, all methanogenic archaea, in spite of their difference in genomic GC content and habitat, show an affinity for higher usage of Cys residues in their proteomes as compared to sulphur-metabolising counterparts. If we consider in terms of frequencies of occurrence, the Cys usages are almost double in case of methanogens [47]. Asp is more abundant in methanogens than the other group, whereas Leu is used more in sulphur metabolisers.

The highest and the lowest values of amino acids in the available orders of methanogens and sulphur metabolisers are presented in Table 1. Frequencies of certain residues, namely Ile, Asn, Lys, cys, Trp, Ala etc., exhibit two-to-three-fold variations within the dataset. Overall the proteomes of sulphur metabolisers are richer in aromatic amino acid residues, for example, Phe finds its highest value in thermococcales, Tyr is mostly used in sulfolobales and $\operatorname{Trp}$ has highest usage in thermoproteales.

Table 1 Highest and lowest values of amino acids in the methanogenic and sulphur metabolising orders

\begin{tabular}{lllll}
\hline $\begin{array}{l}\text { Order with the } \\
\text { highest value }\end{array}$ & $\begin{array}{l}\text { Max } \\
\text { value (\%) }\end{array}$ & $\begin{array}{l}\text { Amino } \\
\text { acid }\end{array}$ & $\begin{array}{l}\text { Min } \\
\text { value (\%) }\end{array}$ & $\begin{array}{l}\text { Order with the } \\
\text { lowest value }\end{array}$ \\
\hline Thermococcales & 4.40 & Phe & 2.88 & Methanopyrales \\
\hline Methanococcales & 9.92 & Ile & 4.81 & Methanopyrales \\
\hline Methanobacteriales & 5.88 & Asn & 1.92 & Methanopyrales \\
\hline Methanococcales & 9.49 & Lys & 3.99 & Methanopyrales \\
\hline Sulfolobales & 4.74 & Tyr & 2.82 & Methanopyrales \\
\hline Methanocellales & 3.00 & Met & 1.65 & Methanopyrales \\
\hline Desulfurococcales & 10.97 & Leu & 8.65 & Methanocellales \\
\hline Methanopyrales & 10.55 & Val & 6.81 & Methanococcales \\
\hline Methanosarcinales & 6.83 & Ser & 4.64 & Methanopyrales \\
\hline Methanomicrobiales & 6.07 & Thr & 4.48 & Desulfurococcales \\
\hline Methanomicrobiales & 2.01 & His & 1.32 & Sulfolobales \\
\hline Methanomicrobiales & 2.87 & Gln & 1.41 & Methanopyrales \\
\hline Methanobacteriales & 6.18 & Asp & 4.39 & Thermoproteales \\
\hline Methanopyrales & 9.99 & Glu & 6.18 & $\begin{array}{l}\text { Unclassified } \\
\text { methanogen RC-I }\end{array}$ \\
\hline Methanomicrobiales & 1.34 & Cys & 0.56 & Thermococcales \\
\hline Thermoproteales & 1.35 & Trp & 0.66 & Methanococcales \\
\hline Methanopyrales & 8.33 & Arg & 3.35 & Methanococcales \\
\hline Methanopyrales & 5.48 & Pro & 3.39 & Methanococcales \\
\hline
\end{tabular}




\section{Correspondence analysis on amino acid usage with two groups}

Figure 5a represents the positions of all 51 organisms in the plane originated by the first two principal axes generated by Correspondence Analysis (COA) on all the amino acid usage. The first two axes explain $63.98 \%$ and $15.44 \%$ variability respectively, contributing a total of $79.42 \%$ to the total variation in the dataset. Unlike the cluster analysis, COA on amino acid usage segregates the two metabolising groups - the methanogenic and the sulphur metabolising organisms - along the second axis of the plane. There are, however, few exceptions. M.fer, M.jan

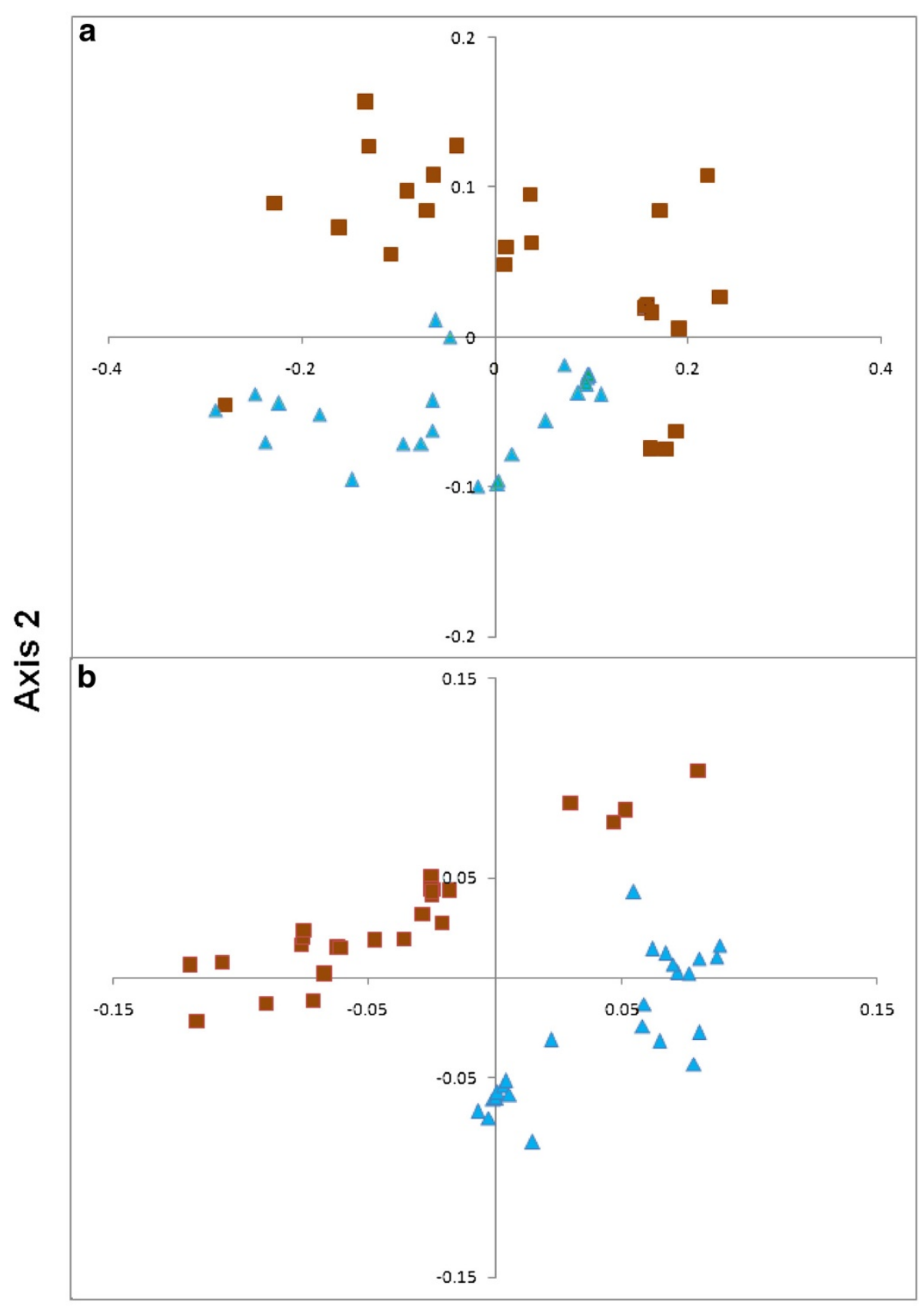

Axis 1

Methanogen $\triangle$ Sulphur metaboliser

Figure 5 Plot of all the methanogens and the sulphur metabolisers in the plane of the first two axes generated by the correspondence analysis on a) all amino acid usage and b) unbiased amino acid usage. 
and M.vul are placed in the fourth quadrant with other sulphur metabolisers and M.kan is placed in the third quadrant. $M$. kandleri, being a unique methane-producing archaeon known to date with dual adaptation to high temperature and high salinity, is marked with its conspicuous presence within the sulphur metabolising group instead of the group of methanogens [46,48]. A strong negative correlation between the first axis and the genomic GC content of the organisms have been observed with $\mathrm{r}=-0.97, \mathrm{p}<10-5$, revealing the fact that the most promising variable contributing to the total variation in the entire dataset is the GC content of the organisms. The second axis shows a negative correlation with the isoelectric point of the proteomes with $\mathrm{r}=-0.71, \mathrm{p}<10^{-5}$.

Correspondence analysis on the unbiased amino acid usage dataset segregates the two types of organisms diagonally, i.e. the variable which decides the segregation should have almost similar correlation with both the axes (Figure 5b). The first two axes explain $63.47 \%$ of the total variation as a whole. Since the genomic GCbias has hardly any influence on the usage of these amino acid residues, significant correlations of isoelectric point have been observed with both the axes(with $r=0.63$ and -0.60 respectively).
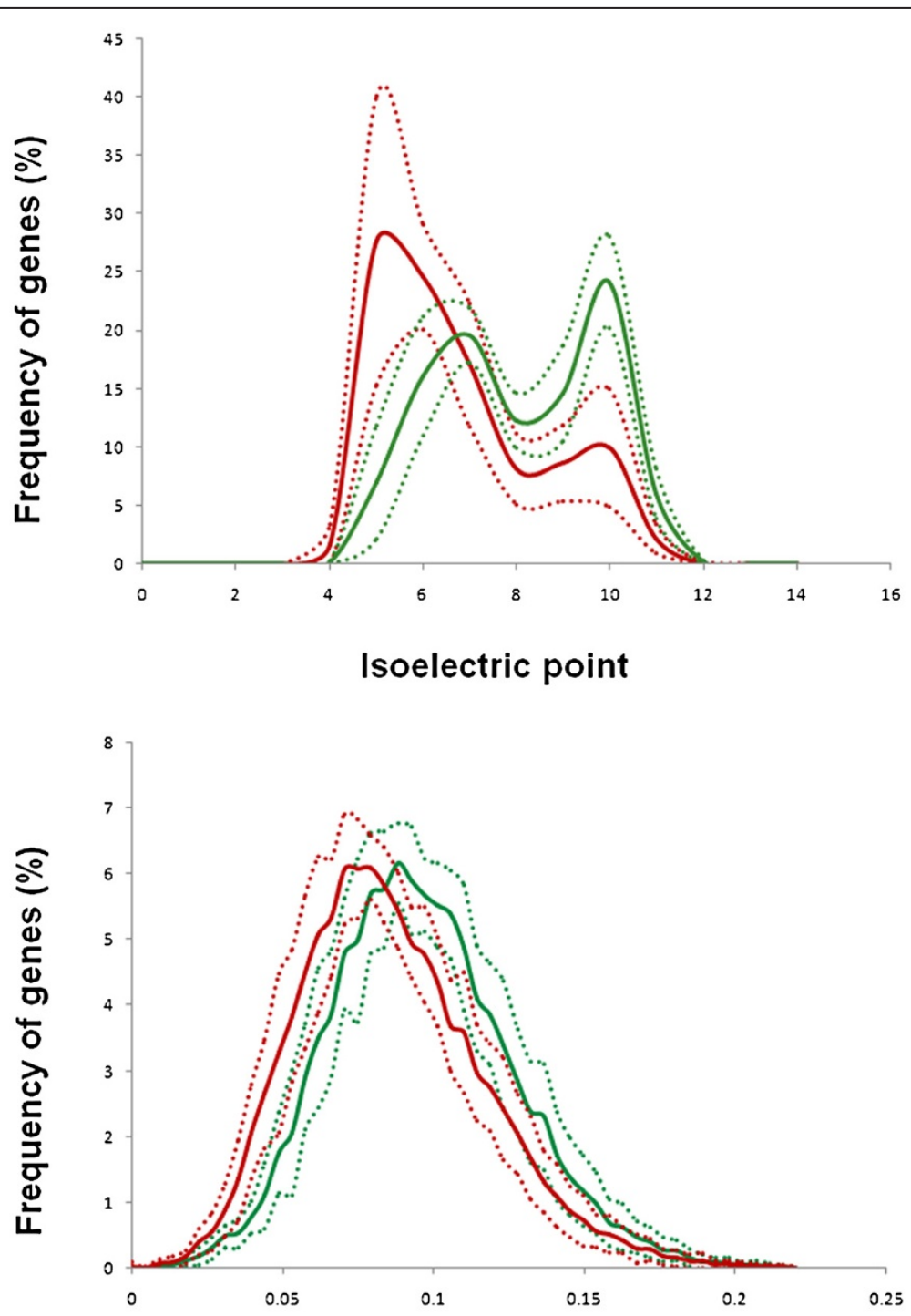

Aromaticity 


\section{Comparative analysis of physico-chemical features of proteomes}

Among all the traditional amino acid usage indices variables, the isoelectric point distribution of the two groups show the most significant difference in them. Figure 6a shows the comparisons between the isoelectric point distributions of the two groups. Previously in Figure 2, the lineage-specific distribution of isoelectric points was presented, where distinct trends in pI profiles was observed in different archaeal phyla. But Figure 6a delineates the overall differences in $\mathrm{pI}$ profiles in two groups of proteomes of distinct metabolic traits, which reveals that methanogens, in general, have more acidic proteomes than their sulphur metabolising counterparts. The plot shows the average percentage number of genes \pm the standard deviations in each pI range in both the cases. It is well established that thermophilic organisms are in general, characterised by basic proteomes [34,42,49]. Since all the sulphur metabolisers available to date are thermophilic/hyperthermophilic in nature, one could argue that the isoelectric point distribution of their proteomes is a mere reflection of their temperature adaptation. But if that was the case, we should have obtained basic proteomes in case of thermophilic/hyperthermophilic methanogenic organisms also, but this is not true. On the contrary, all the methanogenic organisms irrespective of their temperature adaptation individually show the trend of having acidic proteomes (data not shown). Methanogenic proteomes also exhibit relatively low usage of aromatic residues as compared to the sulphur-metabolising archaea (Figure 6b). This observation is in good agreement with the earlier proposition by Das et al. which suggested that higher occurrences of positively charged residues and aromatic residues may facilitate cation-pi interactions in thermophilic/hyperthermophilic proteomes [34].

\section{Surface charge distribution}

Since the distinct patterns in the average isoelectric point distribution in methano gens and sulphur-metabolisers points towards the overall acidic and basic natures of the proteomes of these two groups of archaea respectively, we further wanted to compare the surface charge distribution of orthologous proteins from these two groups. Figure 7 shows the surface charge distribution of the protein glyceraldehyde-3-phosphate-dehydrogenase, from two different organisms, S.solfataricus $P 2$ as a representative from sulphur metabolising group and $M$. jannaschii DSM 2661 from methanogenic group, with sequence similarity of $67 \%$. To avoid the effect of thermal adaptation on the surface charge distribution patterns, both of these thermophilic organisms are consciously selected. Genomic GC contents are also quite similar in both of these organisms. The selection of this particular protein is justified by the fact that it is the only protein whose crystal structures are available for both of these groups [50,51]. The surface charge view has been generated by the program MOLMOL [52], as described in the Materials \& Methods section. Both the organisms are hyperthermophilic and hence should have positive charges on the surfaces, as reported earlier for proteins from other hyperthermophilic microbes $[34,42,49]$. But as can be seen from Figure 7, the protein from M. jannaschii DSM 2661 contains more negatively charged residues on its surface, whereas its $S$. solfataricus $P 2$ ortholog have more positively charged residues on the surface. This observation re-confirms the

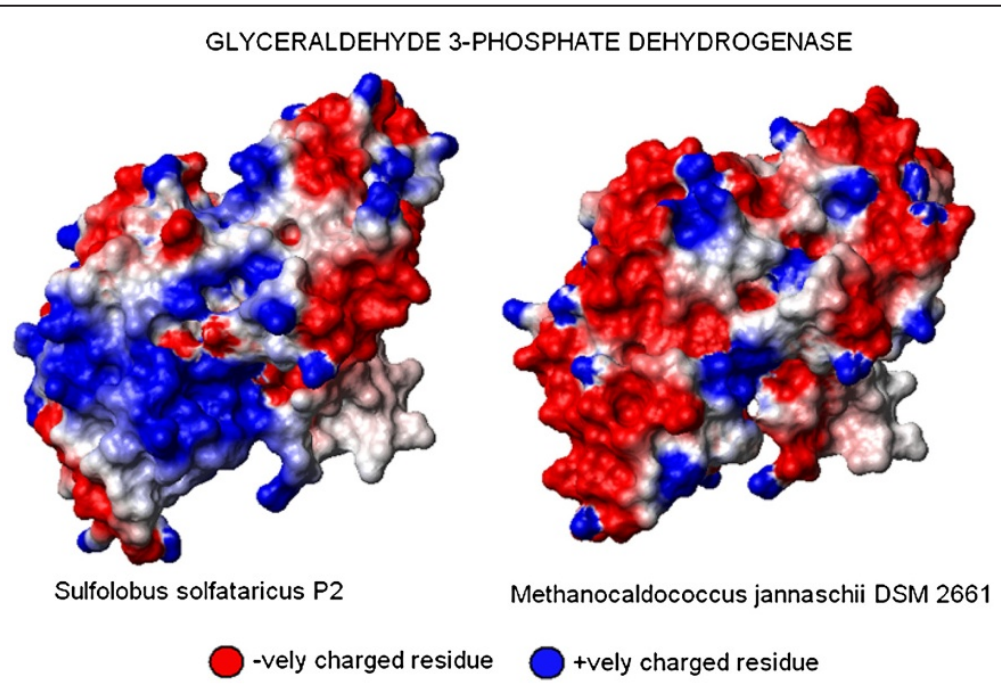

Figure 7 Surface charge distribution pattern of the protein glyceraldehydes-3-phosphate- dehydrogenase in M.jan (methanogen) and S.sol (sulphur metaboliser). 
findings on the isoelectric point distribution in two groups of organisms under study.

\section{Amino acid substitution in orthologous sequences}

Since comparative studies on isoelectric po int distribution and surface charge distribution in proteins of methanogenic and sulphur-metabolising archaea have revealed a clear trend in higher usage of acidic residues in the former group of species (Figure $6 \& 7$ ), it is tempting to examine the general trend, if any, in the amino acid substitution patterns between the orthologous proteins from members of these two different groups. Among all the organisms chosen for this study, we selected two organisms from two groups such that their thermal adaptations, genomic GC contents, genome sizes, and the phylum they are placed in are identical. M.del and T.onn suited well (Additional file 3) for analysing amino acid substitution in their orthologous sequences.

Thus, a distinct trend in the resultant substitution patterns across the orthologs from these organisms can be attributed to the differences in their metabolic traits. The amino acid sequences of these orthologous genes were aligned using ClustalW and the amino acid replacements are arranged in a $20 \times 20$ matrix using
Substitution Pattern Analysis Software Tool (SPAST), a program in $\mathrm{C}++$, developed in-house [53]. Frequencies of all possible amino acid replacements (i.e. $(20 \times 19) /$ $2=190$ possible pairs of replacements) between the orthologous protein sequences were determined in the direction from the methanogenic archaea M.del to the sulphur metabolising archaea T.onn, following the method reported by Paul et al., described in details in the Materials \& Methods section [37].

The prominent trends observed in such replacements are as follows: i) Cyst ines of $\mathrm{M}$. del proteins are replaced by various residues in their $\mathrm{T}$. onn orthologs, ii) different residues of M.del proteins are substituted by aromatic residues, especially by $\operatorname{Trp}$ in $\mathrm{T}$. onn orthologs, iii) Ser and Asp of M.del sequences are replaced by other residues in $\mathrm{T}$. onn sequences. T.onn proteins also show a tendency of acquiring Lys in lieu of other residues. Thus, there is a prevale nce of overall gain in Lys as well as in aromatic residues, especially Trp, and loss in Cys, Ser and Asp in the sulphur-metaboliser T. onn, as compared to the methanogen M.del. A careful scrutiny of the left panel of Table 2 also reveals that the $\mathrm{T}$. onn proteins show a trend of gaining charged residues, not only the positively charged Lys, but also the

Table 2 Top 20 amino acid pairs displaying highest bias in terms of differences and ratios in number of forward (methanogens $\rightarrow$ sulphur metaboliser) and reverse (sulphur metaboliser $\rightarrow$ methanogen) replacements in 213 orthologous proteins from M.del to T. onn

\begin{tabular}{|c|c|c|c|c|c|c|c|c|c|}
\hline \multicolumn{10}{|c|}{ Amino acid replacements between 213 orthologous proteins of M.del \& T. onn } \\
\hline \multicolumn{5}{|c|}{ Most biased in gain } & \multicolumn{5}{|c|}{ Most biased in ratio } \\
\hline Pair & Ratio & Fwd. No & Rev. No. & Gain & Pair & Ratio & Fwd. No. & Rev. No. & Gain \\
\hline$R \rightarrow K$ & $1.97^{\# \#}$ & 871 & 443 & 428 & $\mathrm{Q} \rightarrow \mathrm{W}$ & $8.00^{*}$ & 8 & 1 & 7 \\
\hline $\mathrm{E} \rightarrow \mathrm{K}$ & $2.03^{\# \#}$ & 615 & 303 & 312 & $C \rightarrow M$ & $7.50^{* *}$ & 15 & 2 & 13 \\
\hline$\overline{D \rightarrow E}$ & $1.58^{\# \#}$ & 723 & 458 & 265 & $\mathrm{H} \rightarrow \mathrm{W}$ & $6.00^{* *}$ & 18 & 3 & 15 \\
\hline$S \rightarrow K$ & $3.91^{\# \#}$ & 274 & 70 & 204 & $C \rightarrow I$ & $6.00^{\# \#}$ & 30 & 5 & 25 \\
\hline $\mathrm{D} \rightarrow \mathrm{K}$ & $2.84^{\# \#}$ & 281 & 99 & 182 & $C \rightarrow S$ & $4.75^{\# \#}$ & 38 & 8 & 30 \\
\hline$S \rightarrow A$ & $1.65^{\# \#}$ & 442 & 268 & 174 & $\mathrm{C} \rightarrow \mathrm{N}$ & $4.67^{* *}$ & 14 & 3 & 11 \\
\hline $\mathrm{S} \rightarrow \mathrm{E}$ & $2.41^{\# \#}$ & 296 & 123 & 173 & $\mathrm{D} \rightarrow \mathrm{W}$ & $4.33^{*}$ & 13 & 3 & 10 \\
\hline $\mathrm{I} \rightarrow \mathrm{L}$ & $1.20^{\#}$ & 838 & 701 & 137 & $\mathrm{R} \rightarrow \mathrm{W}$ & $4.25^{\# \#}$ & 34 & 8 & 26 \\
\hline $\mathrm{R} \rightarrow \mathrm{E}$ & $1.40^{\# \#}$ & 436 & 312 & 124 & $\mathrm{~S} \rightarrow \mathrm{K}$ & $3.91^{\# \#}$ & 274 & 70 & 204 \\
\hline$M \rightarrow L$ & $1.45^{\# \#}$ & 379 & 262 & 117 & $C \rightarrow A$ & $3.83^{\# \#}$ & 92 & 24 & 68 \\
\hline$G \rightarrow K$ & $2.22^{\# \#}$ & 182 & 82 & 100 & $M \rightarrow W$ & $3.60^{* *}$ & 18 & 5 & 13 \\
\hline $\overrightarrow{T \rightarrow V}$ & $1.48^{\# \#}$ & 275 & 186 & 89 & $C \rightarrow V$ & $3.55^{\# \#}$ & 71 & 20 & 51 \\
\hline $\overrightarrow{A \rightarrow K}$ & $1.77^{\# \#}$ & 188 & 106 & 82 & $C \rightarrow G$ & $3.50 * *$ & 21 & 6 & 15 \\
\hline $\mathrm{L} \rightarrow \mathrm{F}$ & $1.30^{* *}$ & 328 & 252 & 76 & $\mathrm{~S} \rightarrow \mathrm{W}$ & $3.50^{*}$ & 14 & 4 & 10 \\
\hline$M \rightarrow I$ & $1.51^{\# \#}$ & 204 & 135 & 69 & $D \rightarrow Y$ & $3.24^{\# \# \#}$ & 55 & 17 & 38 \\
\hline $\mathrm{C} \rightarrow \mathrm{A}$ & $3.83^{\# \#}$ & 92 & 24 & 68 & $\mathrm{C} \rightarrow \mathrm{T}$ & 3.20\# & 32 & 10 & 22 \\
\hline$S \rightarrow T$ & $1.33^{* *}$ & 275 & 207 & 68 & $\mathrm{C} \rightarrow \mathrm{F}$ & $3.17^{* *}$ & 19 & 6 & 13 \\
\hline $\mathrm{D} \rightarrow \mathrm{N}$ & 1.43\# & 216 & 151 & 65 & $\mathrm{D} \rightarrow \mathrm{K}$ & $2.84^{\# \#}$ & 281 & 99 & 182 \\
\hline $\mathrm{T} \rightarrow \mathrm{L}$ & $1.62^{\# \#}$ & 165 & 102 & 63 & $\mathrm{~V} \rightarrow \mathrm{W}$ & $2.70^{* *}$ & 27 & 10 & 17 \\
\hline $\overrightarrow{H \rightarrow Y}$ & $2.21^{\# \#}$ & 106 & 48 & 58 & $\mathrm{I} \rightarrow \mathrm{W}$ & $2.44^{*}$ & 22 & 9 & 13 \\
\hline
\end{tabular}


negatively charged residue Glu in place of uncharged residues of their M.del orthologs.

\section{COGs category distribution in the two groups}

The average COGs distribution profiles of different orders/classes of the archaeal domain, along with those of $E$. coli and yeast, have already been depicted in Figure 3. Here we intend to examine the distribution of different functional COGs categories at the level of individual proteomes of the methanogenic and the sulphur metabolising groups of archaea. Wide variations in COGs distribution patterns have been observed among members of methanomicrobia and sulfolobales. Appreciable intra-class/order variations have also been observed among the members of methanobacteria, desulfurococcales and thermoproteales for certain COGs categories, while for methanococci and thermococci, such variations are not so apparent (Figure 8).

Among different COGs categories, certain categories like J (translation, ribosomal structure \& biogenesis), L (replication, recombination \& repair), M (cell wall/membrane biogenesis), $\mathrm{C}$ (energy production \& conversion) etc. exhibit more intra-species divergences within a specific class/order, while the categories like $\mathrm{U}$ (intracellular trafficking, secretion \& vesicular transport), V (defence mechanism), $\mathrm{O}$ (post translational modifications, protein turnover, chaperons) or Q (secondary metabolite biosynthesis, transport and catabolism) show little var iation within or even across different taxonomic orders. Interestingly enough, all three orders under the class thermoproteales of crenaerchaeota, namely sulfolobales, desulfurococcales and thermoproteales exhibit, in general, appreciable intra-order inter-species variations in frequencies of occurrences for most of the COGs categories pertaining to metabolism, but not for the COGs categories under Cellular Processes \& Signaling. Such intra-class variations in frequencies are relatively less, in general, in cases of euryarchaeal organisms, both for metabolism and cellular processes \& signaling COGs categories (Figure 8, left panel).

\section{Identification of core, but exclusive COGs of methanogens}

The next objective of this endeavour is to identify the niche-specific COGs, if any, in two metabolic groups of archaea under study. To this end, all annotated COGs IDs from the 25 methanogenic archaea of the current dataset are enlisted and searched against one another to get the core methanogenic COGs IDs. Each of these core COGs are then searched against the individual COGs contents of 26 sulphur metabolising archaea under examination. In this way we have identified 22 unique COGs, which are present in all methanogens,

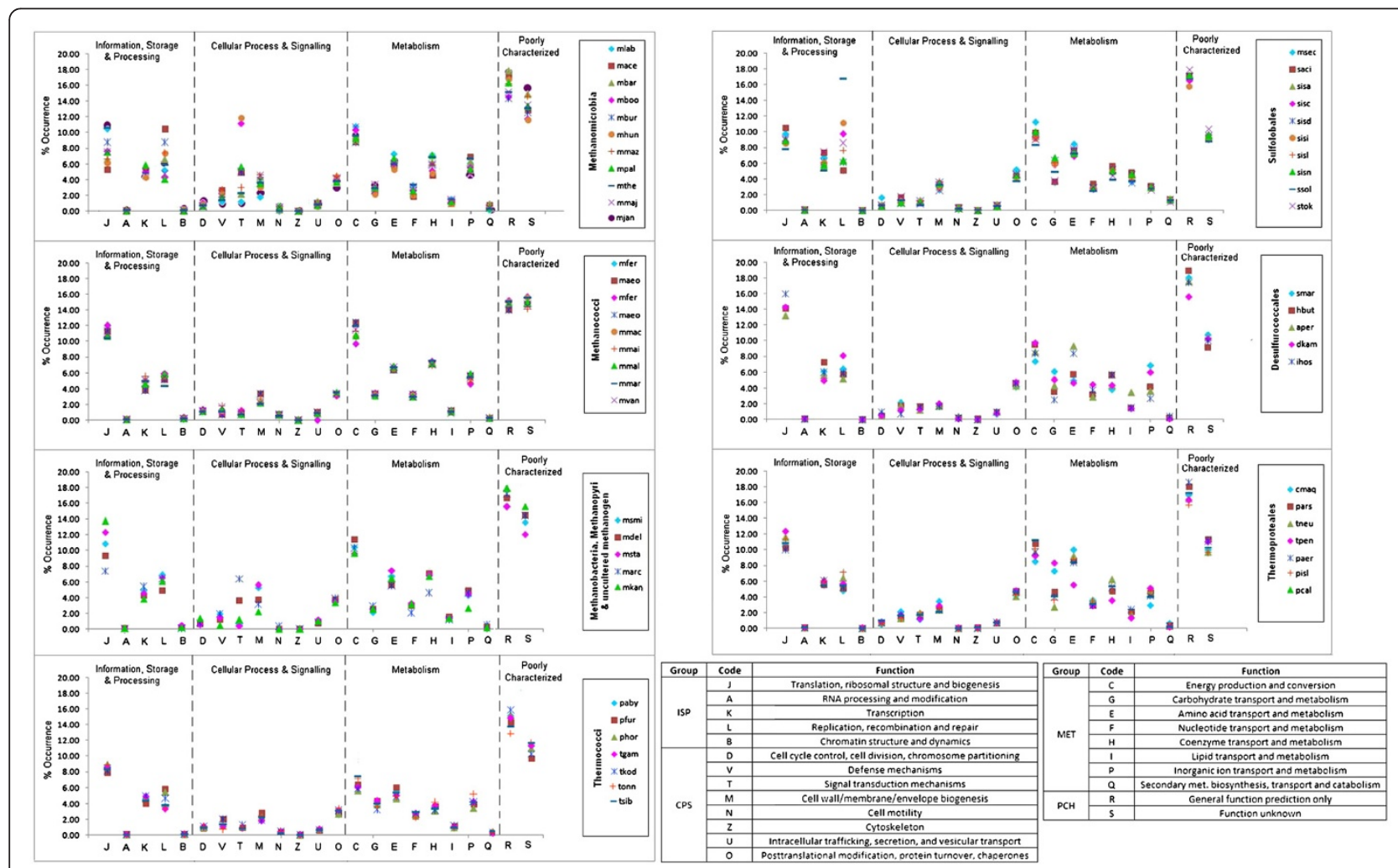

Figure 8 Plot of percentage occurrence of COGs categories in different groups of methanogens and sulphur metabolisers. 
but not in any of the sulphur-metabolisers of the dataset. A further search confirmed that these 22 COGs are not present in any other archaeal proteome under study. Table 3 enlists these methanogen-specific proteins as well as the domains found in their sequences. A reverse search for COGs present in all sulphur-metabolising species, but not in any of the methanogens, does not yield any exclusive protein.

\section{Estimation of COGs shared mutually between distinct groups of methanogens and sulphur metaboliser}

In order to understand the evolutionary relationships between different classes of methanogens and different orders of sulphur metabolisers, the distribution of COGs in those groups of archaea have been examined. Figure 9a \& 9b depict four-variable venn diagrams for the respective groups of methanogens and sulphur metabolisers. The total number of COGs IDs for each group is given within a parenthesis. In case of methanogenic group, the number of exclusive COGs for the class methanococci, methanopyri, methanobacteria and methanomicrobia are 47, 189, 68 and 34 respectively. For the other group, the numbers for the order desulfurococcales, thermoproteales, archaeoglobales and sulfolobales are 42, 16, 842 and 217 respectively. All the methanogenic groups contain a core group of 488 COGs, whereas all the sulphur metabolisers share a core group of 196 COGs (Additional file 4). A further investigation into the categories of the core COGs revealed that apart from J, R and S, methanogens have $\mathrm{E}$ and $\mathrm{H}$, whereas sulphur counterparts have $\mathrm{K}$ and $\mathrm{L}$ respectively among their top five COGs categories (for COGs category, see Additional file 2). So the methanogens have more number of common COGs of the group metabolism and the sulphur counterparts have more number of information storage and processing COGs common in them.

\section{Conclusion}

The present study gives an account of amino acid usage, physico-chemical features and COG repertoire of 69 archaeal species of varying GC-content, habitats, respiratory habits and metabolism. Amino acid usage pattern in archaea, in general, is dominated by the genomic GCcontent, but in some cases niche-specialisation overrules the GC-bias. For amino acids having no GC-bias at their codon levels, environmental factors like oxygen requirement or temperature adaptation appear to be the

Table 3 Methanogen-specific COGs ID and their descriptions

\begin{tabular}{|c|c|c|c|}
\hline COG ID & Description & Domains found on TIGR & Domains found on Pfam \\
\hline COG4058 & Methyl coenzyme $M$ reductase, alpha subunit & \multirow[t]{4}{*}{ met-coenzyme $M$ reductase } & \multirow[t]{4}{*}{ met-coenzyme $M$ reductase } \\
\hline COG4054 & Methyl coenzyme M reductase, beta subunit & & \\
\hline COG4056 & Methyl coenzyme $M$ reductase, subunit $C$ & & \\
\hline COG4055 & Methyl coenzyme $M$ reductase, subunit $D$ & & \\
\hline $\operatorname{COG} 2710$ & $\begin{array}{l}\text { Nitrogenase molybdenum-iron protein, } \\
\text { alpha and beta chains }\end{array}$ & methanogenesis marker protein 13 & oxidored-nitr \\
\hline COG1348 & Nitrogenase subunit NifH (ATPase) & $\mathrm{NifH}$ & Fer4-NifH \\
\hline COG4008 & Predicted metal-binding transcription factor & methanogenesis marker protein 9 & \multirow[t]{3}{*}{ Not found } \\
\hline COG4070 & $\begin{array}{l}\text { Predicted peptidyl-prolyl cis-trans } \\
\text { isomerase (rotamase), cyclophilin family }\end{array}$ & methanogenesis marker protein 3 & \\
\hline COG4002 & Predicted phosphotransacetylase & methanogenesis marker protein 4 & \\
\hline COG4032 & Predicted thiamine-pyrophosphate-binding protein & sulfopyruvate decarboxylase & TPP enzyme \\
\hline COG2144 & Selenophosphate synthetase-related proteins & methanogenesis marker protein 2 & AIRS \\
\hline COG4063 & Tetrahydromethanopterin S-methyltransferase, subunit A & \multirow[t]{5}{*}{ Methyl transferase (Mtr) } & \multirow[t]{5}{*}{ Methyl transferase (Mtr) } \\
\hline COG4062 & Tetrahydromethanopterin S-methyltransferase, subunit B & & \\
\hline COG4061 & Tetrahydromethanopterin S-methyltransferase, subunit C & & \\
\hline COG4060 & Tetrahydromethanopterin S-methyltransferase, subunit D & & \\
\hline COG4059 & Tetrahydromethanopterin S-methyltransferase, subunit E & & \\
\hline COG4050 & Uncharacterized protein conserved in archaea & methanogenesis marker protein 5 & \multirow{6}{*}{$\begin{array}{l}\text { Domain of unknown } \\
\text {-function (DUF) }\end{array}$} \\
\hline COG4051 & Uncharacterized protein conserved in archaea & methanogenesis marker protein 17 & \\
\hline COG4065 & Uncharacterized protein conserved in archaea & methanogenesis marker protein 14 & \\
\hline COG4014 & Uncharacterized protein conserved in archaea & $* * *$ & \\
\hline COG4029 & Uncharacterized protein conserved in archaea & methanogenesis marker protein 6 & \\
\hline COG4052 & $\begin{array}{l}\text { Uncharacterized protein related to methyl coenzyme } \\
M \text { reductase subunit } C\end{array}$ & methanogenesis marker protein 7 & \\
\hline
\end{tabular}




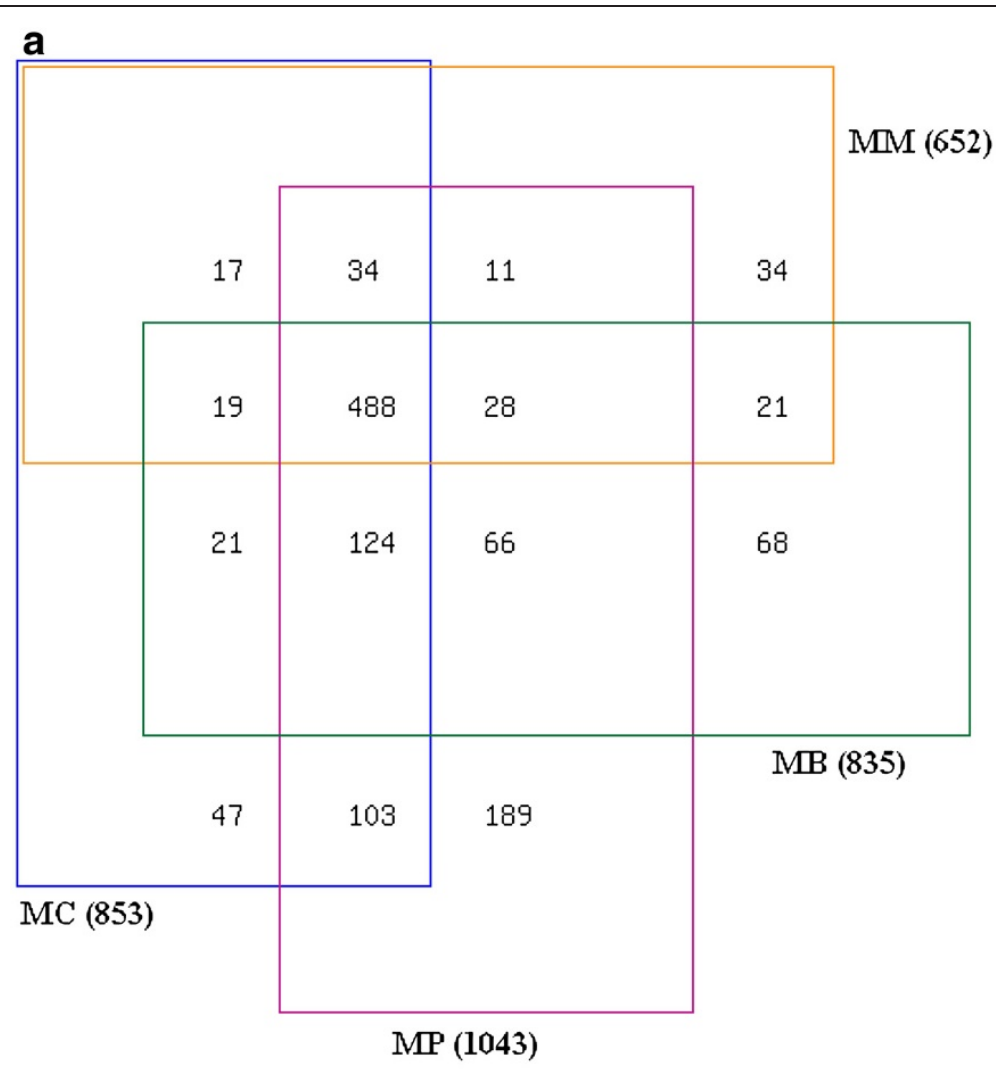

b

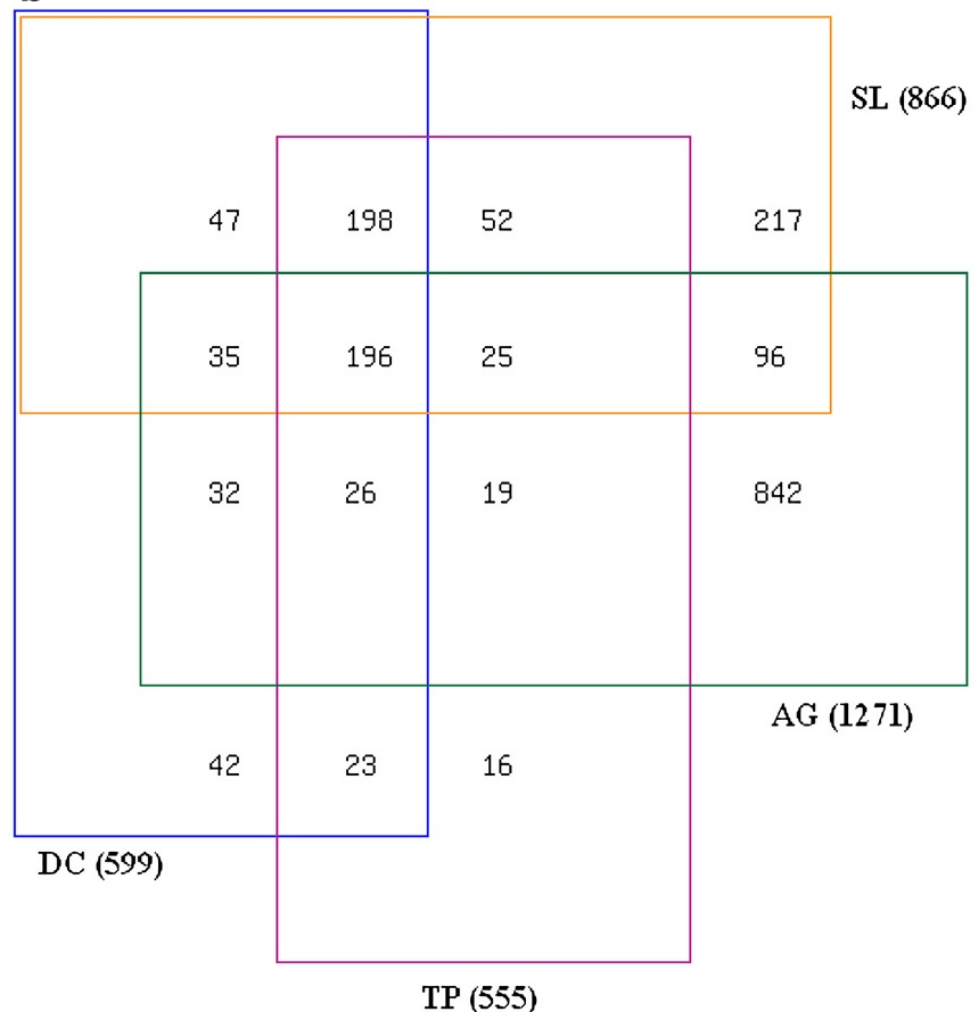

Figure 9 (See legend on next page.) 
primary selection forces. Among the physico-chemical parameters, the overall charge profile and aromaticity of proteins seem to modulate or be modulated by the metabolic traits and/or niche adaptation of the respective species. All methanogenic proteomes, irrespective of their temperature or salinity adptation, are relatively acidic and have higher usage of Cys, while the proteomes of sulphur metabolisers are more basic and aromatic in nature. The atypical (acidic) nature of the thermophilic archaeon $K$. cryptofilum is surprising and demands further investigation in future. So far as COGs repertoire is concerned, crenarchaeal organisms display higher intra-order variations as compared to euryarchaeal counterparts, especially for the proteins involved in metabolism, probably because the divergence of sulphur reduction pathways from those of sulphur oxidation. There are 22 COGs, which are found in all methanogenic archaea under study, not in any other archaea. No such core COGs could be found exclusively within sulphur-metabolising groups.

Identification of distinct trends in amino acid usage, physicochemical properties and COG distribution profiles in methanogens and sulphur-metabolisers, aerobic and anaerobic archaea or korarchaeota and nanoarchaeota point towards the diverse evolutionary strategies for niche specialisation in the archaeal world. Characterisation of such niche-specific features may have farreaching implications of metagenomic or biotechnological perspectives.

\section{Method}

\section{Sequence retrieval}

The complete genome sequences and the predicted protein coding sequences of 69 (all the fully sequenced archaea available by the year 2009) archaea have been downloaded from NCBI GenBank. In order to reduce the sampling errors, the annotated ORFs having less than 100 codons in every genome have been excluded from the analysis. Additional file 1 and Additional file 3 show the basic information about all the archaea under study and about the two studied groups.

\section{Amino acid usage}

Relative amino acid usage frequencies for each organism have been calculated from CODONW [54]. Heat map represents the pictorial version of amino acid frequencies all the organisms, where the colour gradient from red to green in every column shows the increasing values of abundance for a particular amino acid.

\section{Cluster analysis and correspondence analysis on amino} acid usage

To find out the inter-proteomic differences between organisms, the correspondence analysis and the cluster analysis on amino acid composition are carried out using STATISTICA (version 6.0) for all organisms [55]. Correspondence analysis has been done for the two groups of organisms viz. sulphur metabolisers and methanogens. This analysis generates a series of orthogonal axes with each subsequent axis explaining decreasing amount of contribution to the total variation in the dataset.

\section{Indices used to identify the amino acid usage pattern}

To identify the major factors influencing the amino acid usage we calculated the average hydrophobicity (Gravy Score), aromaticity, aliphatic index, instability index and isoelectric point distribution for every organism. We observed significant variation of isoelectric point distribution in case of all the organisms and aromaticity distribution pattern along with the pI distribution in case of the two studied groups, hence we present here the same.

\section{Surface charge distribution}

The surface charge distributions are mapped onto the predicted surface using the program MOLMOL [52]. The protein used here is glyceraldehyde-3-phosphatedehydrogenase from both the methanogenic and the sulphur metabolising archaea viz. M. jannaschii DSM 2661 and S.solfataricus P2 respectively (PDB ID 2YYY and $1 \mathrm{~B} 7 \mathrm{G})$.

\section{Amino acid exchange bias with orthologous sequences}

Orthologous sequences between M.del and T.onn are taken using the tBlastx program [56]. Orthologs are defined as those with more than or equal to $40 \%$ similarities and less than $20 \%$ difference in length and e value $\leq 1 \mathrm{E}-10$. The amino acid sequences of 213 orthologous genes are aligned using the pairwise alignment program ClustalW [57] and the amino acid replacements are obtained in the form of a matrix, using a program developed in-house in Visual Basic [53]. Under unbiased conditions, the ratio of forward to reverse substitutions is expected to be 1:1 for each pair of residues. To test this hypothesis, the observed and expected numbers (based on a 1:1 ratio) are recorded for each pair of residues and the chi-square test is applied to assess the significance of the directional bias, if any, at significance levels of 10-3 to 10-6. For a 
given pair of amino acids, the 'forward' direction exhibited the more common of the two replacements in the conversion of methanogenic to the sulphur metabolising proteins. To assess the significance of the directional bias, if any, replacement values are compared by $2 \times 2$ contingency tables having one degree of freedom. For each pair of replacements, the first and second rows of the contingency table represented the number of replacements from one particular residue (say, i) to another (say, j) of the pair and the total count of the remaining replacements (say, k) from the residue $\mathrm{i}$ (where $\mathrm{k} \neq \mathrm{j}$ ) respectively.

\section{COGs (cluster of orthologous groups of proteins) distribution}

The predicted COGs annotations for each protein of all the organisms have been done with the help of WebMGA server [58]. Only those proteins are taken for which COGs IDs have been annotated and hence other proteins are excluded. The percentage number of COGs category present in every organism has been calculated. For a better resolution of the gene possession of every phylum, the methanogens and sulphur metabolisers have been further divided into their corresponding classes and orders respectively.

\section{Additional files}

\section{Additional data file1: Detail information about all archaea under} study.

Additional data file2: Details of COGs category.

Additional data file3: Details of all sulphur metabolising and methanogenic archaea under study.

Additional data file4: Descriptions about the methanogenic and sulphur metabolising core COGs.

\section{Abbreviations}

COGs: Cluster of Orthologous Group of proteins; COA: Correspondence Analysis; SPAST: Substitution Pattern Analysis Software Tool.

\section{Competing interests}

The authors declare that they have no competing interests.

\section{Acknowledgements}

We are grateful to Dr. Sandip Paul, Microbiology, University of Washington, for thoughtful and constructive suggestions during the progress of the study and for critical reading of the manuscript. We thankfully acknowledge the infrastructural support obtained from the Bioinformatics Centre of this institute. This work was supported by Council of Scientific and Industria Research (Project no. CMM 017). ARC is supported by Senior Research Fellowships from Council of Scientific and Industrial Research, India.

\section{Authors' contribution}

ARC contributed to the development of work plan, carried out sequence analysis and other statistical/computational studies and drafted the manuscript. CD conceived the work, participated in the design and coordination of the study and revised the manuscript critically for important intellectual content. Both the authors read and approved the final manuscript.
Received: 25 January 2012 Accepted: 12 June 2012

Published: 12 June 2012

\section{References}

1. Woese CR, Fox GE: Phylogenetic structure of the prokaryotic domain: the primary kingdoms. Proc Natl Acad Sci 1977, 74(11):5088.

2. Makarova KS, Koonin EV: Comparative genomics of Archaea: how much have we learned in six years, and what's next. Genome Biol 2003, 4(8):115.

3. Woese CR: The archaeal concept and the world it lives in: a retrospective. Photosynth Res 2004, 80(1):361-372.

4. Woese CR: The birth of the Archaea: a personal retrospective. in Archaea, Evolution, Physiology \& Molecular Biology. USA; Blackwell Publishing Ltd: Garrett, RA, Klenk HP, Malden, MA; 2007:1.

5. Woese CR, Magrum LJ, Fox GE: Archaebacteria. J Mol Evol 1978, 11(3):245-252.

6. Woese CR, Kandler O, Wheelis ML: Towards a natural system of organisms: proposal for the domains Archaea, Bacteria, and Eucarya. Proc Natl Acad Sci 1990, 87(12):4576.

7. Woese CR, Gupta R: Are archaebacteria merely derived â€ prokaryotesâ $€^{\mathrm{T} M}$ ? Nature 1981, 289(5793):95-96.

8. Pace NR, Olsen GJ, Woese CR: Ribosomal RNA phylogeny and the primary lines of evolutionary descent. Cell 1986, 45(3):325.

9. Zillig W: Comparative biochemistry of Archaea and Bacteria. Curr Opin Genet Dev 1991, 1(4):544-551.

10. Fox GE, Stackebrandt E, Hespell RB, Gibson J, Maniloff J, Dyer TA, Wolfe RS, Balch WE, Tanner RS, Magrum LJ, et al: The phylogeny of prokaryotes. Science 1980, 209(4455):457-463.

11. Auchtung TA, Takacs-Vesbach CD, Cavanaugh CM: 16 S rRNA phylogenetic investigation of the candidate division "Korarchaeota". Appl Environ Microbiol 2006, 72(7):5077-5082.

12. Brochier-Armanet C, Boussau B, Gribaldo S, Forterre P: Mesophilic Crenarchaeota: proposal for a third archaeal phylum, the Thaumarchaeota. Nat Rev Microbiol 2008, 6(3):245-252.

13. Elkins JG, Podar M, Graham DE, Makarova KS, Wolf Y, Randau L, Hedlund BP, Brochier-Armanet C, Kunin V, Anderson I, et al: A korarchaeal genome reveals insights into the evolution of the Archaea. Proc Natl Acad Sci U S A 2008, 105(23):8102-8107.

14. Huber H, Hohn MJ, Rachel R, Fuchs T, Wimmer VC, Stetter KO: A new phylum of Archaea represented by a nanosized hyperthermophilic symbiont. Nature 2002, 417(6884):63-67.

15. Molloy S: Archaea: Thaumarchaeota go it alone. Nat Rev Microbiol 2011, 9(12):832.

16. Nealson K: A Korarchaeote yields to genome sequencing. Proc Natl Acad Sci U S A 2008, 105(26):8805-8806.

17. Schouten S, Hopmans EC, Baas M, Boumann H, Standfest S, Konneke M, Stahl DA, Sinninghe Damste JS: Intact membrane lipids of "Candidatus Nitrosopumilus maritimus," a cultivated representative of the cosmopolitan mesophilic group I Crenarchaeota. Appl Environ Microbiol 2008, 74(8):2433-2440

18. Keeling PJ, Charlebois RL, Ford Doolittle W: Archaebacterial genomes: eubacterial form and eukaryotic content. Curr Opin Genet Dev 1994, 4(6):816-822.

19. Lykke-Andersen J, Aagaard C, Semionenkov M, Garrett RA: Archaeal introns: splicing, intercellular mobility and evolution. Trends Biochem Sci 1997, 22(9):326-331.

20. Watanabe Y, Yokobori S, Inaba T, Yamagishi A, Oshima T, Kawarabayasi Y, Kikuchi H, Kita K: Introns in protein-coding genes in Archaea. FEBS Lett 2002, 510(1-2):27-30.

21. Lecompte O, Ripp R, Thierry JC, Moras D, Poch O: Comparative analysis of ribosomal proteins in complete genomes: an example of reductive evolution at the domain scale. Nucleic Acids Res 2002, 30(24):5382-5390

22. Londei P: Evolution of translational initiation: new insights from the archaea. FEMS Microbiol Rev 2005, 29(2):185-200.

23. Werner F, Eloranta JJ, Weinzierl RO: Archaeal RNA polymerase subunits $F$ and P are bona fide homologs of eukaryotic RPB4 and RPB12. Nucleic Acids Res 2000, 28(21):4299-4305.

24. Gaspin C, Cavaille J, Erauso G, Bachellerie JP: Archaeal homologs of eukaryotic methylation guide small nucleolar RNAs: lessons from the Pyrococcus genomes. J Mol Biol 2000, 297(4):895-906.

25. Reeve JN, Sandman K, Daniels CJ: Archaeal histones, nucleosomes, and transcription initiation. Cell 1997, 89(7):999-1002. 
26. Hartung S, Hopfner KP: Lessons from structural and biochemical studies on the archaeal exosome. Biochem Soc Trans 2009, 37(Pt 1):83-87.

27. Tornabene TG, Langworthy TA: Diphytanyl and dibiphytanyl glycerol ether lipids of methanogenic archaebacteria. Science 1979, 203(4375):51.

28. Woese CR: Bacterial evolution. Microbiol Mol Biol Rev 1987, 51(2):221.

29. Bapteste E, Brochier C, Boucher Y: Higher-level classification of the Archaea: evolution of methanogenesis and methanogens. Archaea 2005 1(5):353-363.

30. Forterre P, Gribaldo S, Gadelle D, Serre MC: Origin and evolution of DNA topoisomerases. Biochimie 2007, 89(4):427-446.

31. Ishino Y, Komori K, Cann IK, Koga Y: A novel DNA polymerase family found in Archaea. J Bacteriol 1998, 180(8):2232-2236.

32. Cavicchioli R, Curmi PM, Saunders N, Thomas T: Pathogenic archaea: do they exist? Bioessays 2003, 25(11):1119-1128.

33. Lepp PW, Brinig MM, Ouverney CC, Palm K, Armitage GC, Relman DA: Methanogenic Archaea and human periodontal disease. Proc Natl Acad Sci U S A 2004, 101(16):6176-6181.

34. Das S, Paul S, Bag SK, Dutta C: Analysis of Nanoarchaeum equitans genome and proteome composition: indications for hyperthermophilic and parasitic adaptation. BMC Genom 2006, 7:186

35. Egorova K, Antranikian G: Industrial relevance of thermophilic Archaea. Curr Opin Microbiol 2005, 8(6):649-655

36. Leveque E, Janecek S, Haye B, Belarbi A: Thermophilic archaeal amylolytic enzymes. Enzym Microb Technol 2000, 26(1):3-14.

37. Paul S, Bag SK, Das S, Harvill ET, Dutta C: Molecular signature of hypersaline adaptation: insights from genome and proteome composition of halophilic prokaryotes. Genome Biol 2008, 9(4):70.

38. Waters E, Hohn MJ, Ahel I, Graham DE, Adams MD, Barnstead M, Beeson KY, Bibbs L, Bolanos R, Keller M, et al: The genome of Nanoarchaeum equitans: insights into early archaeal evolution and derived parasitism. Proc Natl Acad Sci U S A 2003, 100(22):12984-12988.

39. Zierenberg RA, Adams MWW, Arp AJ: Life in extreme environments: Hydrothermal vents. Proc Natl Acad Sci 2000, 97(24):12961.

40. Fukuchi S, Yoshimune K, Wakayama M, Moriguchi M, Nishikawa K: Unique amino acid composition of proteins in halophilic bacteria. J Mol Biol 2003, 327(2):347-357.

41. Singh SK, Verma P, Ramaiah N, Chandrashekar AA, Shouche YS: Phylogenetic diversity of archaeal $16 \mathrm{~S}$ rRNA and ammonia monooxygenase genes from tropical estuarine sediments on the central west coast of India. Res Microbiol 2010, 161(3):177-186.

42. Kreil DP, Ouzounis CA: Identification of thermophilic species by the amino acid compositions deduced from their genomes. Nucleic Acids Res 2001, 29(7):1608-1615.

43. Tatusov RL, Fedorova ND, Jackson JD, Jacobs AR, Kiryutin B, Koonin EV, Krylov DM, Mazumder R, Mekhedov SL, Nikolskaya AN, et al: The COG database: an updated version includes eukaryotes. BMC Bioinforma 2003, 4:41.

44. Ventosa A, Societies FoEM, Sevilla Ud: Halophilic microorganisms. Springer; 2004.

45. Lobry JR, Chessel D: Internal correspondence analysis of codon and amino-acid usage in thermophilic bacteria. J Appl Genet 2003, 44(2):235-262

46. Slesarev Al, Mezhevaya KV, Makarova KS, Polushin NN, Shcherbinina OV, Shakhova W, Belova GI, Aravind L, Natale DA, Rogozin IB: The complete genome of hyperthermophile Methanopyrus kandleri AV19 and monophyly of archaeal methanogens. Proc Natl Acad Sci 2002, 99(7):4644.

47. Klipcan L, Frenkel-Morgenstern M, Safro MG: Presence of tRNA-dependent pathways correlates with high cysteine content in methanogenic Archaea. Trends Genet 2008, 24(2):59-63.

48. Burggraf S, Stetter K, Rouviere P, Woese C: Methanopyrus kandleri: an archaeal methanogen unrelated to all other known methanogens. Syst Appl Microbiol 1991, 14:346.

49. Zeldovich KB, Berezovsky IN, Shakhnovich El: Protein and DNA sequence determinants of thermophilic adaptation. Plos Comput Biol 2007 3(1):62-72.

50. Isupov MN, Fleming TM, Dalby AR, Crowhurst GS, Bourne PC, Littlechild JA: Crystal structure of the glyceraldehyde-3-phosphate dehydrogenase from the hyperthermophilic archaeon Sulfolobus solfataricus. J Mol Biol 1999, 291(3):651-660.

51. Malay AD, Bessho Y, Ellis MJ, Antonyuk SV, Strange RW, Hasnain SS, Shinkai A, Padmanabhan B, Yokoyama S: Structure of glyceraldehyde-3-phosphate dehydrogenase from the archaeal hyperthermophile

Methanocaldococcus jannaschii. Acta Crystallographica Section F-Struct Biol Crystallization Comm 2009, 65:1227-1233.

52. Koradi R, Billeter M, Wüthrich K: MOLMOL: a program for display and analysis of macromolecular structures. J Mol Graph 1996, 14(1):51-55.

53. Bag SK, Paul S, Ghosh S, Dutta C: Reverse Polarization in Amino acid and Nucleotide Substitution Patterns Between Humanâ€"Mouse Orthologs of Two Compositional E xtrema. DNA Res 2007, 14(4):141-154.

54. Peden J: CodonW, Trinity College.; 1997.

55. Statsoft I: STATISTICA for Windows (Computer program manual). OK Statisoft: Tulsa; 1995.

56. Altschul SF, Gish W, Miller W, Myers EW, Lipman DJ: Basic Local Alignment Search Tool. J Mol Biol 1990, 215(3):403-410.

57. Thompson JD, Gibson TJ, Higgins DG: Multiple sequence alignment using ClustalW and ClustalX. Curr Protocol Bioinformatics 2002, 23:2.3.1-2.3.22.

58. Wu ST, Zhu ZW, Fu LM, Niu BF, Li WZ: WebMGA: a customizable web server for fast metagenomic sequence analysis. BMC Genom 2011, 12:444.

doi:10.1186/1471-2164-13-236

Cite this article as: Roy Chowdhury and Dutta: A pursuit of lineagespecific and niche-specific proteome features in the world of archaea. BMC Genomics 2012 13:236

\section{Submit your next manuscript to BioMed Central and take full advantage of:}

- Convenient online submission

- Thorough peer review

- No space constraints or color figure charges

- Immediate publication on acceptance

- Inclusion in PubMed, CAS, Scopus and Google Scholar

- Research which is freely available for redistribution

Submit your manuscript at www.biomedcentral.com/submit
C Biomed Central 